\title{
ON THE SPATIAL BEHAVIOR IN THERMOELASTICITY WITHOUT ENERGY DISSIPATION
}

\author{
M. Ciarletta ${ }^{1}$ and S. Chiriţă ${ }^{2}$ \\ ${ }^{1}$ DIIMA, University of Salerno, Fisciano, SA, Italy \\ ${ }^{2}$ Faculty of Mathematics, A1. I. Cuza University of Iaşi, Iaşi, Romania
}

In the present paper we consider a prismatic cylinder occupied by an anisotropic homogeneous compressible linear thermoelastic material that is subject to zero body force and heat supply and zero displacement and thermal displacement on the lateral boundary. The motion is induced by a harmonic time-dependent displacement-thermal displacement specified pointwise over the base. We establish some spatial estimates for some appropriate cross-sectional measures associated with the transient and steady state solutions that describe how they evolves with respect to the axial distance to the excited base. For the transient solutions a domain of influence is observed inside which a measure of the solution decays exponentially with respect to the axial variable, while outside of the domain the solution vanishes. For the harmonic in time vibrations we establish spatial estimates describing how the amplitude of vibration evolves with respect to the axial variable, provided the frequency is lower than a critical value. All results are established for the thermoelastic materials whose constitutive coefficients satisfy strong ellipticity conditions.

Keywords: Spatial behavior; Strong elliptic elasticity tensor; Thermoelasticity without energy dissipation; Transient and steady state solutions

\section{INTRODUCTION}

In the literature concerning thermal effects in continuum mechanics there are developed several parabolic and hyperbolic theories for describing the heat conduction. The hyperbolic theories are also called theories of second sound and there the flow of heat is modelled with finite propagation speed, in contrast to the classical model based on the Fourier's law leading to infinite propagation speed of heat signals. A review of these theories is presented in the articles by Chandrasekharaiah [1] and Hetnarski and Ignaczak [2, 3].

A new thermoelastic theory without energy dissipation has been proposed by Green and Naghdi [4]. This thermomechanical theory of deformable media introduces the so-called thermal displacement and uses a general entropy balance as postulated in [5]. By the procedure of Green and Naghdi [6], the reduced energy

Received 24 January 2009; accepted 31 March 2009.

The author SC acknowledges support from the Romanian Ministry of Education and Research, CNCSIS Grant code ID-401, Contract no. 15/28.09.2007.

Address correspondence to S. Chiriţă, Faculty of Mathematics, A1. I. Cuza University of Iaşi, Blvd. Carol I, nr. 11, Iaşi 700506, Romania. E-mail: schirita@uaic.ro 
equation is regarded as an identity for all thermodynamical processes and places some restrictions on the functional forms of the dependent constitutive variables. The theory is illustrated in detail in the context of flow of heat in a rigid solid, with particular reference to the propagation of thermal waves at finite speed. The linearized formulation allows the transmission of heat flow as thermal waves at finite speed and the evolution equations are fully hyperbolic.

The linear theory of thermoelasticity without energy dissipation for homogeneous and isotropic materials was employed by Nappa [7] and Quintanilla [8] in order to obtain spatial energy bounds and decay estimates for the transient solutions in connection with the problem in which a thermoelastic body is deformed subject to boundary and initial data and body supplies having a compact support, provided positive definiteness assumptions are supposed upon the constitutive coefficients. Moreover, we have to mention that Chandrasekharaiah [9] proves uniqueness of solutions, Ieşan [10] establishes continuous dependence results, while Quintanilla [11] studies the question of existence. Further results of structural stability and decay type are given by Quintanilla [12, 13]. Quintanilla and Straughan [14] used logarithmic convexity and Lagrange identity arguments to yield uniqueness and growth without requiring sign definiteness of the constitutive coefficients, while Quintanilla and Straughan [15] derive energy bounds for a class of non-standard problems in which the initial data are given as a combination of data at initial time and at a later time.

The present paper is concerned with the study of the spatial behavior of the transient and harmonic in time solutions for the initial and boundary value problems associated with the linear thermoelasticity theory without dissipation energy for anisotropic materials. We derive some differential inequalities for certain cross-sectional integrals and integration leads to estimates describing how these integrals evolve with respect to the axial variable. To achieve this goal we use a method based on the technique developed by Flavin et al. [16] for the classical elastic problem and later developed by Chiriţă and Quintanilla [17] and Chiriţă and Ciarletta [18] (for dynamic problems) and Chiriţă [19] (for steady-state solutions). However, here we use an idea developed by Chiriţă [20] for linear thermoelasticity of anisotropic materials with a strong elliptic elasticity tensor. Such a method establishes differential inequalities for the selected measures which after integration provides estimates for spatial evolution, provided the strong ellipticity of the constitutive coefficients is assumed.

\section{NOTATION AND BASIC THEORY}

Throughout this section we assume that the region $\Omega$ is occupied by a homogeneous and anisotropic thermoelastic material with a center of symmetry at each point. In what follows we will consider the dynamic theory of thermoelasticity without energy dissipation as described in $[4,6]$.

The governing equations of the linear theory of anisotropic and homogeneous thermoelasticity without energy dissipation are given by the evolution equations $[4,6]$

$$
\begin{aligned}
& S_{j i, j}+\rho b_{i}=\rho \ddot{u}_{i} \\
& \rho \dot{\eta}=\frac{\rho}{\theta_{0}} r-q_{i, i}
\end{aligned}
$$


in $\Omega \times(0, \infty)$, the constitutive equations

$$
\begin{gathered}
S_{i j}=C_{i j k l} e_{k l}-M_{i j} \theta \\
\rho \eta=M_{i j} e_{i j}+\frac{c}{\theta_{0}} \theta \\
q_{i}=-\frac{1}{\theta_{0}} K_{i j} \beta_{j}
\end{gathered}
$$

in $\bar{\Omega} \times[0, \infty)$, the geometrical equations

$$
e_{i j}=\frac{1}{2}\left(u_{i, j}+u_{j, i}\right)
$$

in $\bar{\Omega} \times[0, \infty)$, and the law of heat flow

$$
\dot{\beta}_{i}=\theta_{, i}
$$

in $\bar{\Omega} \times[0, \infty)$. Here $u_{i}$ are the components of the displacement vector, $\theta$ is the temperature variation from the uniform reference temperature $\theta_{0}, e_{i j}$ are the components of the strain tensor, $\beta_{i}$ are the components of the thermal displacement gradient vector, $S_{i j}$ are the components of the stress tensor, $q_{i}$ are the components of the entropy-heat flux vector, $\eta$ is the entropy density per unit mass and $b_{i}$ represents the components of the external body force vector and $r$ is the external rate of supply of heat per unit mass. Furthermore, $\rho$ is the constant density mass, $C_{i j k l}, M_{i j}, c$ and $K_{i j}$ are the constant constitutive coefficients satisfying the following symmetries

$$
C_{i j k l}=C_{k l i j}=C_{j i k l}, \quad M_{i j}=M_{j i}, \quad K_{i j}=K_{j i}
$$

and the subscripts $i, j, k, l$ take values $1,2,3$ and summation is implied by index repetition. Moreover, a superposed dot denotes differentiation with respect to time and a subscript comma indicates partial differentiation. The specific Helmholtz free energy $\psi$ is given by

$$
\rho \psi=\frac{1}{2} C_{i j k l} e_{i j} e_{k l}-M_{i j} e_{i j} \theta-\frac{c}{2 \theta_{0}} \theta^{2}+\frac{1}{2 \theta_{0}} K_{i j} \tau_{, i} \tau_{, j}
$$

while the specific internal energy $\epsilon$ is given by

$$
\rho \epsilon=\frac{1}{2} C_{i j k l} e_{i j} e_{k l}+\frac{c}{2 \theta_{0}} \theta^{2}+\frac{1}{2 \theta_{0}} K_{i j} \tau_{, i} \tau_{, j}
$$

where $\tau$ is the thermal displacement related to the temperature variation by relation

$$
\dot{\tau}=\theta
$$


In view of the relations (1)-(6) and (9) we can express the basic field equations in terms of the displacements $u_{i}$ and the thermal displacement $\tau$ as

$$
\begin{aligned}
\left(C_{r s m n} u_{m, n}-M_{r s} \dot{\tau}\right)_{, r}+\rho b_{s} & =\rho \ddot{u}_{s} \\
\frac{1}{\theta_{0}}\left(K_{r s} \tau_{, s}\right)_{, r}-M_{r s} \dot{u}_{r, s}+\frac{\rho}{\theta_{0}} r & =\frac{c}{\theta_{0}} \ddot{\tau}
\end{aligned}
$$

in $\Omega \times(0, \infty)$. Clearly, the present theory permits propagation of heat as thermal waves at finite speed.

To the above governing equations we adjoin the initial conditions

$$
u_{s}(x, 0)=u_{s}^{0}(x), \quad \dot{u}_{s}(x, 0)=\dot{u}_{s}^{0}(x), \quad \tau(x, 0)=\tau^{0}(x), \quad \theta(x, 0)=\theta^{0}(x), \quad x \in \Omega
$$

and the boundary conditions

$$
u_{s}(x, t)=\tilde{u}_{s}(x, t), \quad \tau(x, t)=\tilde{\tau}(x, t), \quad(x, t) \in \partial \Omega \times(0, \infty)
$$

where $u_{s}^{0}(x), \dot{u}_{s}^{0}(x), \tau^{0}(x), \theta^{0}(x), \tilde{u}_{s}(x, t)$ and $\tilde{\tau}(x, t)$ are prescribed smooth functions. Other types of boundary conditions can be also considered. We assume that the given data are sufficiently smooth to assure the existence of a solution $\left(u_{i}, \tau\right)$ satisfying the above initial and boundary value problem.

For later convenience we have to note that for a positive definite specific internal energy $\epsilon$ there are satisfied the following conditions

$$
\begin{gathered}
C_{r s m n} \xi_{r s} \xi_{m n}>0 \text { for all non-zero } \xi_{r s}=\xi_{s r} \\
c>0, \quad K_{r s} \xi_{r} \xi_{s}>0 \text { for all non-zero vector } \xi_{r}
\end{gathered}
$$

However, for the analysis of the present study we assume the strong ellipticity of the elasticity tensor $C_{r s m n}$, a hypothesis that is weaker than the corresponding positive definite conditions expressed by (13). So, in what follows we assume that

$$
C_{r s m n} \xi_{r} \xi_{m} \zeta_{s} \zeta_{n}>0 \text { for all non-zero vectors }\left(\xi_{1}, \xi_{2}, \xi_{3}\right),\left(\zeta_{1}, \zeta_{2}, \zeta_{3}\right)
$$

Our results will be exemplified for thermoelastic materials with rhombic symmetry. Using a standard notation for the non-zero components of the elasticity tensor, $C_{r s m n}$, the strong ellipticity condition (14) becomes

$$
\begin{aligned}
& c_{11} \xi_{1}^{2} \zeta_{1}^{2}+c_{22} \xi_{2}^{2} \zeta_{2}^{2}+c_{33} \xi_{3}^{2} \zeta_{3}^{2}+c_{66}\left(\xi_{1} \zeta_{2}+\xi_{2} \zeta_{1}\right)^{2}+c_{55}\left(\xi_{1} \zeta_{3}+\xi_{3} \zeta_{1}\right)^{2} \\
& \quad+c_{44}\left(\xi_{2} \zeta_{3}+\xi_{3} \zeta_{2}\right)^{2}+2 c_{12} \xi_{1} \zeta_{1} \xi_{2} \zeta_{2}+2 c_{13} \xi_{1} \zeta_{1} \xi_{3} \zeta_{3}+2 c_{23} \xi_{2} \zeta_{2} \xi_{3} \zeta_{3}>0
\end{aligned}
$$

for all non-zero vectors $\left(\xi_{1}, \xi_{2}, \xi_{3}\right),\left(\zeta_{1}, \zeta_{2}, \zeta_{3}\right)$. It was shown in [21] that the condition (15) is equivalent to the following conditions

$$
c_{11}>0, \quad c_{22}>0, \quad c_{33}>0, \quad c_{44}>0, \quad c_{55}>0, \quad c_{66}>0
$$




$$
\begin{aligned}
& -2 c_{66}+\kappa_{3}^{i} \sqrt{c_{11} c_{22}}<c_{12}<\kappa_{3}^{s} \sqrt{c_{11} c_{22}} \\
& -2 c_{44}+\kappa_{1}^{i} \sqrt{c_{22} c_{33}}<c_{23}<\kappa_{1}^{s} \sqrt{c_{22} c_{33}} \\
& -2 c_{55}+\kappa_{2}^{i} \sqrt{c_{33} c_{11}}<c_{13}<\kappa_{2}^{s} \sqrt{c_{33} c_{11}}
\end{aligned}
$$

where $\left(\kappa_{1}^{i}, \kappa_{1}^{s}\right),\left(\kappa_{2}^{i}, \kappa_{2}^{s}\right)$ and $\left(\kappa_{3}^{i}, \kappa_{3}^{s}\right)$ are the two solutions with respect to $x, y$ and $z$ of the equation $x^{2}+y^{2}+z^{2}-2 x y z-1=0$ satisfying $|x|<1,|y|<1,|z|<1$, with $x$ in the set $\left\{\frac{c_{23}}{\sqrt{c_{22} c_{33}}}, \frac{c_{23}+2 c_{44}}{\sqrt{c_{22} c_{33}}}\right\}, y$ in the set $\left\{\frac{c_{13}}{\sqrt{c_{11} c_{33}}}, \frac{c_{13}+2 c_{55}}{\sqrt{c_{11} c_{33}}}\right\}$ and $z$ in the set $\left\{\frac{c_{12}}{\sqrt{c_{11} c_{22}}}, \frac{c_{12}+2 c_{66}}{\sqrt{c_{11} c_{22}}}\right\}$. That means all the points $P(x, y, z)$ with coordinates $x \in\left\{\frac{c_{23}}{\sqrt{c_{22} c_{33}}}, \frac{c_{23}+2 c_{44}}{\sqrt{c_{22} c_{33}}}\right\}, y \in$ $\left\{\frac{c_{13}}{\sqrt{c_{11} c_{33}}}, \frac{c_{13}+2 c_{55}}{\sqrt{c_{11} c_{33}}}\right\}$ and $z \in\left\{\frac{c_{12}}{\sqrt{c_{11} c_{22}}}, \frac{c_{12}+2 c_{66}}{\sqrt{c_{11} c_{22}}}\right\}$ lie inside the domain limited by the surface $S(x, y, z) \equiv x^{2}+y^{2}+z^{2}-2 x y z-1=0,|x|<1,|y|<1,|z|<1$. The form of this domain can be seen in [21].

In the study of the transient solutions we will assume that the constitutive coefficients satisfy the strong ellipticity conditions (16) and (17) and

$$
c>0, \quad K_{33}>0, \quad K_{\alpha \beta} \xi_{\alpha} \xi_{\beta} \geq 0 \text { for all } \xi_{\alpha}
$$

While for the analysis of the harmonic in time solutions we require the positive definiteness of $K_{\alpha \beta}$ instead of the positive semi-definiteness expressed in (18), that is we will assume that (18) is substituted by

$$
c>0, \quad K_{33}>0, \quad K_{\alpha \beta} \xi_{\alpha} \xi_{\beta}>0 \text { for all non-zero } \xi_{\alpha}
$$

\section{TRANSIENT SOLUTIONS}

Let us consider that the region $\Omega$ is a prismatic cylinder $B \subset \mathbb{R}^{3}$ whose bounded uniform cross-section $D \subset \mathbb{R}^{2}$ has piecewise continuously differentiable boundary $\partial D$. The origin of the rectangular Cartesian coordinate system is located in the cylinder's base and the positive $x_{3}$-axis is directed along that of the cylinder. Then the lateral boundary is $\pi=\partial D \times[0, L]$, where $L$ is the length of the cylinder. The cylinder is occupied by a homogeneous and anisotropic linear thermoelastic solid with rhombic symmetry. It is subjected to zero body force and heat supply, zero displacement and thermal displacement on the lateral boundary surface and pointwise specified displacement and thermal displacement over the base. Thus, we are studying the spatial evolution of the solution $\left(u_{i}, \tau\right)$ of the initial boundary value problem $\mathscr{P}$ defined by the differential system

$$
\begin{aligned}
\rho \ddot{u}_{1}= & c_{11} u_{1,11}+c_{66} u_{1,22}+c_{55} u_{1,33}+\left(c_{12}+c_{66}\right) u_{2,21}+\left(c_{13}+c_{55}\right) u_{3,31} \\
& -M_{11} \dot{\tau}_{, 1}-M_{12} \dot{\tau}_{, 2} \\
\rho \ddot{u}_{2}= & \left(c_{12}+c_{66}\right) u_{1,12}+c_{66} u_{2,11}+c_{22} u_{2,22}+c_{44} u_{2,33}+\left(c_{23}+c_{44}\right) u_{3,32} \\
& -M_{12} \dot{\tau}_{, 1}-M_{22} \dot{\tau}_{, 2} \\
\rho \ddot{u}_{3}= & \left(c_{13}+c_{55}\right) u_{1,13}+\left(c_{23}+c_{44}\right) u_{2,23}+c_{55} u_{3,11}+c_{44} u_{3,22}+c_{33} u_{3,33}-M_{33} \dot{\tau}_{, 3} \\
\frac{c}{\theta_{0}} \ddot{\tau}= & \frac{1}{\theta_{0}}\left(K_{11} \tau_{, 11}+2 K_{12} \tau_{, 12}+K_{22} \tau_{, 22}+K_{33} \tau_{, 33}\right) \\
& -M_{11} \dot{u}_{1,1}-M_{12}\left(\dot{u}_{1,2}+\dot{u}_{2,1}\right)-M_{22} \dot{u}_{2,2}-M_{33} \dot{u}_{3,3}
\end{aligned}
$$


in $B \times(0, \infty)$, with the initial conditions

$$
u_{s}(x, 0)=0, \quad \dot{u}_{s}(x, 0)=0, \quad \tau(x, 0)=0, \quad \theta(x, 0)=0, \quad x \in B
$$

and the lateral and end boundary conditions

$$
u_{s}(x, t)=0, \quad \tau(x, t)=0, \quad(x, t) \in(\pi \cup D(L)) \times(0, \infty)
$$

and the base boundary conditions

$u_{s}\left(x_{1}, x_{2}, 0, t\right)=\tilde{u}_{s}\left(x_{1}, x_{2}, t\right), \quad \tau\left(x_{1}, x_{2}, 0, t\right)=\tilde{\tau}\left(x_{1}, x_{2}, t\right), \quad\left(x_{1}, x_{2}, t\right) \in D(0) \times(0, \infty)$

When a semi-infinite cylinder will be considered then the boundary condition (22) has to be considered only for $(x, t) \in \pi \times(0, \infty)$.

Properties of solution $\left(u_{i}, \tau\right)$ are established by means of a function related to a cross-sectional integral that enables a differential inequality to be derived. The function considered is defined by

$$
\begin{aligned}
I\left(x_{3}, t\right)=-\int_{0}^{t} \int_{D\left(x_{3}, \sigma\right)}\{ & {\left[c_{55} u_{1,3}+\left(c_{55}-\varkappa_{2}\right) u_{3,1}\right] \dot{u}_{1}+\left[c_{44} u_{2,3}+\left(c_{44}-\varkappa_{1}\right) u_{3,2}\right] \dot{u}_{2} } \\
& +\left[\left(c_{13}+\varkappa_{2}\right) u_{1,1}+\left(c_{23}+\varkappa_{1}\right) u_{2,2}+c_{33} u_{3,3}\right] \dot{u}_{3} \\
& \left.-M_{33} \dot{u}_{3} \dot{\tau}+\frac{1}{\theta_{0}} K_{33} \dot{\tau} \tau_{, 3}\right\} d a d \sigma
\end{aligned}
$$

for $\left(x_{3}, t\right) \in[0, L] \times[0, \infty)$. In the above integral the term $D\left(x_{3}, \sigma\right)$ is used to indicate that relevant quantities are to be evaluated at time $t=\sigma$ over the crosssection whose distance from the origin is $x_{3}$. Moreover, there are the parameters $\varkappa_{1}$ and $\varkappa_{2}$ at our disposal and their values will be defined later.

By direct differentiation with respect to time variable in (24) we obtain

$$
\begin{gathered}
\frac{\partial I}{\partial t}\left(x_{3}, t\right)=-\int_{D\left(x_{3}, t\right)}\left\{\left[c_{55} u_{1,3}+\left(c_{55}-\varkappa_{2}\right) u_{3,1}\right] \dot{u}_{1}+\left[c_{44} u_{2,3}+\left(c_{44}-\varkappa_{1}\right) u_{3,2}\right] \dot{u}_{2}\right. \\
+\left[\left(c_{13}+\varkappa_{2}\right) u_{1,1}+\left(c_{23}+\varkappa_{1}\right) u_{2,2}+c_{33} u_{3,3}\right] \dot{u}_{3} \\
\left.-M_{33} \dot{u}_{3} \dot{\tau}+\frac{1}{\theta_{0}} K_{33} \dot{\tau} \tau_{, 3}\right\} d a
\end{gathered}
$$

Furthermore, by differentiation with respect to $x_{3}$-variable in (24) and then by the use of the basic system (20) and the boundary conditions (22) and the initial conditions (21), we deduce that

$$
\begin{aligned}
-\frac{\partial I}{\partial x_{3}}\left(x_{3}, t\right)= & \frac{1}{2} \int_{D\left(x_{3}, t\right)}\left[\rho \dot{u}_{i} \dot{u}_{i}+\frac{c}{\theta_{0}} \dot{\tau}^{2}+\frac{1}{\theta_{0}}\left(K_{\alpha \beta} \tau_{, \alpha} \tau_{, \beta}+K_{33} \tau_{, 3}^{2}\right)\right] d a \\
& +\frac{1}{2} \int_{D\left(x_{3}, t\right)}\left[c_{11} u_{1,1}^{2}+c_{22} u_{2,2}^{2}+c_{33} u_{3,3}^{2}+c_{66}\left(u_{1,2}^{2}+u_{2,1}^{2}\right)\right. \\
& +c_{55}\left(u_{1,3}^{2}+u_{3,1}^{2}\right)+c_{44}\left(u_{2,3}^{2}+u_{3,2}^{2}\right)+2\left(c_{12}+\varkappa_{3}\right) u_{1,1} u_{2,2}
\end{aligned}
$$




$$
\begin{aligned}
& +2\left(c_{13}+\varkappa_{2}\right) u_{1,1} u_{3,3}+2\left(c_{23}+\varkappa_{1}\right) u_{2,2} u_{3,3}+2\left(c_{66}-\varkappa_{3}\right) u_{1,2} u_{2,1} \\
& \left.+2\left(c_{44}-\varkappa_{1}\right) u_{2,3} u_{3,2}+2\left(c_{55}-\varkappa_{2}\right) u_{1,3} u_{3,1}\right] d a
\end{aligned}
$$

with $\varkappa_{3}$ a positive parameter at our disposal. In the deduction of the relation (26) we have also used the identity

$$
\int_{D\left(x_{3}, t\right)} u_{1,1} u_{2,2} d a=\int_{D\left(x_{3}, t\right)} u_{1,2} u_{2,1} d a
$$

Now, in view of the convexity of the domain limited by the surface $S(x, y, z)=$ $x^{2}+y^{2}+z^{2}-2 x y z-1=0,|x|<1,|y|<1,|z|<1$, we can choose the parameters $\varkappa_{1} \in\left[0,2 c_{44}\right], \varkappa_{2} \in\left[0,2 c_{55}\right]$ and $\varkappa_{3} \in\left[0,2 c_{66}\right]$ so that the point $P(x, y, z)$, with coordinates $x=\frac{c_{23}+\varkappa_{1}}{\sqrt{c_{22} c_{33}}}, y=\frac{c_{13}+\varkappa_{2}}{\sqrt{c_{11} c_{33}}}, z=\frac{c_{12}+\varkappa_{3}}{\sqrt{c_{11} c_{22}}}$, lies inside the region limited by the surface $S(x, y, z)$. Then, the Sylvester criterium provides a straightforward way toward conditions of positive definiteness of the quadratic form in terms of $u_{r, s}$ :

$$
\begin{aligned}
\Phi\left(u_{r, s}\right)= & c_{11} u_{1,1}^{2}+c_{22} u_{2,2}^{2}+c_{33} u_{3,3}^{2}+c_{66}\left(u_{1,2}^{2}+u_{2,1}^{2}\right)+c_{55}\left(u_{1,3}^{2}+u_{3,1}^{2}\right) \\
& +c_{44}\left(u_{2,3}^{2}+u_{3,2}^{2}\right)+2\left(c_{12}+\varkappa_{3}\right) u_{1,1} u_{2,2}+2\left(c_{13}+\varkappa_{2}\right) u_{1,1} u_{3,3} \\
& +2\left(c_{23}+\varkappa_{1}\right) u_{2,2} u_{3,3}+2\left(c_{66}-\varkappa_{3}\right) u_{1,2} u_{2,1}+2\left(c_{44}-\varkappa_{1}\right) u_{2,3} u_{3,2} \\
& +2\left(c_{55}-\varkappa_{2}\right) u_{1,3} u_{3,1}
\end{aligned}
$$

Moreover, we have

$$
\mu_{m} u_{r, s} u_{r, s} \leq \Phi\left(u_{i, j}\right) \leq \mu_{M} u_{r, s} u_{r, s}
$$

where $\mu_{m}$ and $\mu_{M}$ are the positive minimum and maximum eigenvalues of the positive definite quadratic form $\Phi\left(u_{r},{ }_{s}\right)$. On this basis and by means of the hypothesis expressed by relation (18), we can see that (26) implies

$$
\begin{aligned}
-\frac{\partial I}{\partial x_{3}}\left(x_{3}, t\right) \geq & \frac{1}{2} \int_{D\left(x_{3}, t\right)}\left(\rho \dot{u}_{i} \dot{u}_{i}+\frac{c}{\theta_{0}} \dot{\tau}^{2}+\frac{1}{\theta_{0}} K_{33} \tau_{, 3}^{2}\right) d a \\
& +\frac{1}{2} \int_{D\left(x_{3}, t\right)} \mu_{m} u_{r, s} u_{r, s} d a \geq 0
\end{aligned}
$$

and hence $I\left(x_{3}, t\right)$ is a non-increasing function with respect to $x_{3}$-variable for every $t \in[0, \infty)$. Since the boundary condition (22) together with relation (24) imply that $I(L, t)=0$ for all $t \in[0, \infty)$, it follows then we have

$$
I\left(x_{3}, t\right) \geq I(L, t)=0 \text { for all }\left(x_{3}, t\right) \in[0, L] \times[0, \infty)
$$

Moreover, by direct integration with respect to $x_{3}$ over $\left[x_{3}, L\right]$ into relation (30), we obtain

$$
I\left(x_{3}, t\right) \geq \frac{1}{2} \int_{B\left(x_{3}, t\right)}\left(\rho \dot{u}_{i} \dot{u}_{i}+\frac{c}{\theta_{0}} \dot{\tau}^{2}+\frac{1}{\theta_{0}} K_{33} \tau_{, 3}^{2}\right) d v+\frac{1}{2} \int_{B\left(x_{3}, t\right)} \mu_{m} u_{r, s} u_{r, s} d u \geq 0
$$


where $B\left(x_{3}, t\right)$ is the cylinder $D \times\left[x_{3}, L\right]$. This proves that $I\left(x_{3}, t\right)$ represents a measure for the solution in concern.

Now we write the relation $(25)$ in the form

$$
\begin{aligned}
-\frac{\partial I}{\partial t}\left(x_{3}, t\right)=\int_{D\left(x_{3}, t\right)}\{ & \sqrt{\frac{c_{55}}{\rho}}\left(\frac{1}{\sqrt{c_{55}}}\left[c_{55} u_{1,3}+\left(c_{55}-\varkappa_{2}\right) u_{3,1}\right]\right)\left(\sqrt{\rho} \dot{u}_{1}\right) \\
& +\sqrt{\frac{c_{44}}{\rho}}\left(\frac{1}{\sqrt{c_{44}}}\left[c_{44} u_{2,3}+\left(c_{44}-\varkappa_{1}\right) u_{3,2}\right]\right)\left(\sqrt{\rho} \dot{u}_{2}\right) \\
& +\sqrt{\frac{c_{33}}{\rho}}\left[\frac{c_{13}+\varkappa_{2}}{\sqrt{c_{11} c_{33}}}\left(\sqrt{c_{11}} u_{1,1}\right)\left(\sqrt{\rho} \dot{u}_{3}\right)\right. \\
& \left.\left.+\frac{c_{23}+\varkappa_{1}}{\sqrt{c_{22} c_{33}}}\left(\sqrt{c_{22}} u_{2,2}\right)\left(\sqrt{\rho} \dot{u}_{3}\right)+\left(\sqrt{c_{33}} u_{3,3}\right)\left(\sqrt{\rho} \dot{u}_{3}\right)\right]\right\} d a \\
+\int_{D\left(x_{3}, t\right)} & {\left[-\frac{M_{33} \sqrt{\theta_{0}}}{\sqrt{c \rho}}\left(\sqrt{\rho} \dot{u}_{3}\right)\left(\sqrt{\frac{c}{\theta_{0}}} \dot{\tau}\right)+\sqrt{\frac{K_{33}}{c}}\left(\sqrt{\frac{c}{\theta_{0}}} \dot{\tau}\right)\left(\sqrt{\frac{K_{33}}{\theta_{0}}} \tau_{, 3}\right)\right] d a }
\end{aligned}
$$

and use the inequalities $\left|c_{55}-\varkappa_{2}\right|<c_{55},\left|c_{44}-\varkappa_{1}\right|<c_{44},\left|c_{13}+\varkappa_{2}\right|<\sqrt{c_{11} c_{33}}, \mid c_{23}+$ $\varkappa_{1} \mid<\sqrt{c_{22} c_{33}}$ and the arithmetic-geometric and Schwarz inequalities to obtain the estimate

$$
\begin{aligned}
\left|\frac{\partial I}{\partial t}\left(x_{3}, t\right)\right| \leq & \frac{1}{2} \int_{D\left(x_{3}, t\right)}\left[\frac{1}{\sqrt{\rho}} \max \left(\sqrt{c_{55}}, \sqrt{c_{44}}, 3 \sqrt{c_{33}}+\frac{\left|M_{33}\right| \sqrt{\theta_{0}}}{\sqrt{c}}\right) \rho \dot{u}_{i} \dot{u}_{i}\right. \\
& \left.+\left(\frac{\left|M_{33}\right| \sqrt{\theta_{0}}}{\sqrt{c \rho}}+\sqrt{\frac{K_{33}}{c}}\right) \frac{c}{\theta_{0}} \dot{\tau}^{2}+\sqrt{\frac{K_{33}}{c}} \frac{1}{\theta_{0}} K_{33} \tau_{, 3}^{2}\right] d a \\
& +\frac{1}{2} \int_{D\left(x_{3}, t\right)} \frac{1}{\sqrt{\rho}} \max \left(2 c_{55} \sqrt{c_{55}}, 2 c_{44} \sqrt{c_{44}}, c_{11} \sqrt{c_{33}}, c_{22} \sqrt{c_{33}}, c_{33} \sqrt{c_{33}}\right) \\
& \times u_{r, s} u_{r, s} d a
\end{aligned}
$$

Further, we set

$$
v=\max \left(m_{1}, m_{2}\right)
$$

with

$$
\begin{aligned}
& m_{1}=\max \left(\frac{1}{\sqrt{\rho}} \max \left(\sqrt{c_{55}}, \sqrt{c_{44}}, 3 \sqrt{c_{33}}+\frac{\left|M_{33}\right| \sqrt{\theta_{0}}}{\sqrt{c}}\right), \frac{\left|M_{33}\right| \sqrt{\theta_{0}}}{\sqrt{c \rho}}+\sqrt{\frac{K_{33}}{c}}\right) \\
& m_{2}=\frac{1}{\mu_{m} \sqrt{\rho}} \max \left(2 c_{55} \sqrt{c_{55}}, 2 c_{44} \sqrt{c_{44}}, c_{11} \sqrt{c_{33}}, c_{22} \sqrt{c_{33}}, c_{33} \sqrt{c_{33}}\right)
\end{aligned}
$$

so that the relations (30) and (34) give

$$
\left|\frac{\partial I}{\partial t}\left(x_{3}, t\right)\right| \leq-v \frac{\partial I}{\partial x_{3}}\left(x_{3}, t\right), \quad\left(x_{3}, t\right) \in(0, L) \times[0, \infty)
$$


We proceed now to explore the consequences of the differential inequality (37). To this end we first note that it can be written equivalent as

$$
\begin{aligned}
& \frac{1}{v} \frac{\partial I}{\partial t}\left(x_{3}, t\right)+\frac{\partial I}{\partial x_{3}}\left(x_{3}, t\right) \leq 0, \quad\left(x_{3}, t\right) \in(0, L) \times[0, \infty) \\
& -\frac{1}{v} I\left(x_{3}, t\right)+\frac{\partial I}{\partial x_{3}}\left(x_{3}, t\right) \leq 0, \quad\left(x_{3}, t\right) \in(0, L) \times[0, \infty)
\end{aligned}
$$

Further, we choose $t>0$ and $x_{3} \geq v t$. By setting $x_{3}=v t$ in (38) we obtain

$$
\frac{d}{d t}[I(v t, t)] \leq 0
$$

and hence we have

$$
I(v t, t) \leq I(0,0)=0
$$

On the other hand, since $I\left(x_{3}, t\right)$ is a non-increasing function with respect to $x_{3}$ for every $t \in[0, \infty)$, it follows that for $x_{3} \geq v t$, we have

$$
I\left(x_{3}, t\right) \leq I(v t, t) \leq 0
$$

Consequently, the relations (32) and (42) and the initial conditions (21) imply that

$$
u_{s}(x, t)=0, \quad \tau(x, t)=0 \text { for all } 0 \leq v t \leq x_{3} \leq L
$$

that is, for all $t \in\left[0, \frac{L}{v}\right]$, the whole activity in the cylinder $B(v t, t)$ is vanishing and thus a domain of influence holds in the theory of thermoelasticity without dissipation energy.

We have to outline that such kind of result is valuable for a semi-infinite cylinder yet (that is the case when $L \longrightarrow \infty$ ). To this end we have to note that the result expressed by relation (43) was based on the relation $I(L, t)=0$. For the case of a semi-infinite cylinder it is sufficiently to prove that

$$
I(\infty, t)=\lim _{x_{3} \rightarrow \infty} I\left(x_{3}, t\right)=0
$$

In this aim we fix $t_{0}>0$ and consider $x_{3}^{0} \geq v t_{0}$. If we set $x_{3}=x_{3}^{0}+v\left(t-t_{0}\right)$ into relation (38) then we get

$$
\frac{d}{d x_{3}}\left[I\left(x_{3}, t_{0}+\frac{x_{3}-x_{3}^{0}}{v}\right)\right] \leq 0
$$

and hence, by taking into account that $0 \leq x_{3}=x_{3}^{0}-v t_{0} \leq x_{3}^{0}$ we deduce that

$$
I\left(x_{3}^{0}, t_{0}\right) \leq I\left(x_{3}^{0}-v t_{0}, 0\right)=0
$$


Further, we set $x_{3}=x_{3}^{0}-v\left(t-t_{0}\right)$ in (39) to obtain

$$
\frac{d}{d x_{3}}\left[I\left(x_{3}, t_{0}-\frac{x_{3}-x_{3}^{0}}{v}\right)\right] \leq 0
$$

so that we have

$$
0=I\left(x_{3}^{0}+v t_{0}, 0\right) \leq I\left(x_{3}^{0}, t_{0}\right)
$$

Thus, by making $x_{3}^{0} \rightarrow \infty$ into relations (46) and (48), we get the relation (44) and hence our previous analysis proves that (43) implies that for each $t>0$ the whole activity in the cylinder $B(v t, t)$ is vanishing.

In what follows we proceed to study the spatial behavior of solution in the cylinder $D \times[0, v t], t>0$. To this end we define

$$
\begin{aligned}
J\left(x_{3}, t\right)=-\int_{0}^{t} \int_{D\left(x_{3}, \sigma\right)} e^{-\delta \sigma}\{ & {\left[c_{55} u_{1,3}+\left(c_{55}-\varkappa_{2}\right) u_{3,1}\right] \dot{u}_{1}+\left[c_{44} u_{2,3}+\left(c_{44}-\varkappa_{1}\right) u_{3,2}\right] \dot{u}_{2} } \\
& +\left[\left(c_{13}+\varkappa_{2}\right) u_{1,1}+\left(c_{23}+\varkappa_{1}\right) u_{2,2}+c_{33} u_{3,3}\right] \dot{u}_{3} \\
& \left.-M_{33} \dot{u}_{3} \dot{\tau}+\frac{1}{\theta_{0}} K_{33} \dot{\tau} \tau_{, 3}\right\} d a d \sigma
\end{aligned}
$$

where $\delta$ is a positive parameter at our disposal. We obtain

$$
\begin{aligned}
-\frac{\partial J}{\partial x_{3}}\left(x_{3}, t\right)= & \frac{1}{2} \int_{D\left(x_{3}, t\right)} e^{-\delta t}\left[\rho \dot{u}_{i} \dot{u}_{i}+\frac{c}{\theta_{0}} \dot{\tau}^{2}+\frac{1}{\theta_{0}}\left(K_{\alpha \beta} \tau_{, \alpha} \tau_{, \beta}+K_{33} \tau_{, 3}^{2}\right)\right] d a \\
& +\frac{1}{2} \int_{D\left(x_{3}, t\right)} e^{-\delta t}\left[c_{11} u_{1,1}^{2}+c_{22} u_{2,2}^{2}+c_{33} u_{3,3}^{2}+c_{66}\left(u_{1,2}^{2}+u_{2,1}^{2}\right)\right. \\
& +c_{55}\left(u_{1,3}^{2}+u_{3,1}^{2}\right)+c_{44}\left(u_{2,3}^{2}+u_{3,2}^{2}\right)+2\left(c_{12}+\varkappa_{3}\right) u_{1,1} u_{2,2} \\
& +2\left(c_{13}+\varkappa_{2}\right) u_{1,1} u_{3,3}+2\left(c_{23}+\varkappa_{1}\right) u_{2,2} u_{3,3}+2\left(c_{66}-\varkappa_{3}\right) u_{1,2} u_{2,1} \\
& \left.+2\left(c_{44}-\varkappa_{1}\right) u_{2,3} u_{3,2}+2\left(c_{55}-\varkappa_{2}\right) u_{1,3} u_{3,1}\right] d a \\
& +\frac{\delta}{2} \int_{0}^{t} \int_{D\left(x_{3}, \sigma\right)} e^{-\delta \sigma}\left[\rho \dot{u}_{i} \dot{u}_{i}+\frac{c}{\theta_{0}} \dot{\tau}^{2}+\frac{1}{\theta_{0}}\left(K_{\alpha, \beta} \tau_{, \alpha} \tau_{, \beta}+K_{33} \tau_{, 3}^{2}\right)\right] d a d \sigma \\
& +\frac{\delta}{2} \int_{0}^{t} \int_{D\left(x_{3}, \sigma\right)} e^{-\delta \sigma}\left[c_{11} u_{1,1}^{2}+c_{22} u_{2,2}^{2}+c_{33} u_{3,3}^{2}+c_{66}\left(u_{1,2}^{2}+u_{2,1}^{2}\right)\right. \\
& +c_{55}\left(u_{1,3}^{2}+u_{3,1}^{2}\right)+c_{44}\left(u_{2,3}^{2}+u_{3,2}^{2}\right)+2\left(c_{12}+\varkappa_{3}\right) u_{1,1} u_{2,2} \\
& +2\left(c_{13}+\varkappa_{2}\right) u_{1,1} u_{3,3}+2\left(c_{23}+\varkappa_{1}\right) u_{2,2} u_{3,3}+2\left(c_{66}-\varkappa_{3}\right) u_{1,2} u_{2,1} \\
& \left.+2\left(c_{44}-\varkappa_{1}\right) u_{2,3} u_{3,2}+2\left(c_{55}-\varkappa_{2}\right) u_{1,3} u_{3,1}\right] d a d \sigma
\end{aligned}
$$

where the identity (27) was also used. Moreover, we have

$$
J\left(x_{3}, t\right) \geq \frac{1}{2} \int_{B\left(x_{3}, t\right)} e^{-\delta t}\left(\rho \dot{u}_{i} \dot{u}_{i}+\frac{c}{\theta_{0}} \dot{\tau}^{2}+\frac{1}{\theta_{0}} K_{33} \tau_{, 3}^{2}\right) d v
$$




$$
\begin{aligned}
& +\frac{1}{2} \int_{B\left(x_{3}, t\right)} e^{-\delta t} \mu_{m} u_{r, s} u_{r, s} d v+\frac{\delta}{2} \int_{0}^{t} \int_{B\left(x_{3}, \sigma\right)} e^{-\delta \sigma} \mu_{m} u_{r, s} u_{r, s} d v d \sigma \\
& +\frac{\delta}{2} \int_{0}^{t} \int_{B\left(x_{3}, \sigma\right)} e^{-\delta \sigma}\left(\rho \dot{u}_{i} \dot{u}_{i}+\frac{c}{\theta_{0}} \dot{\tau}^{2}+\frac{1}{\theta_{0}} K_{33} \tau_{, 3}^{2}\right) d v d \sigma \geq 0
\end{aligned}
$$

Further, we can obtain the first-order differential inequality

$$
\frac{\delta}{v} J\left(x_{3}, t\right)+\frac{\partial J}{\partial x_{3}}\left(x_{3}, t\right) \leq 0 \text { for all } t \geq 0, x_{3} \leq v t
$$

which by direct integration gives the spatial estimate

$$
0 \leq J\left(x_{3}, t\right) \leq J(0, t) e^{-\frac{\delta}{v} x_{3}} \text { for all } t \geq 0, \quad x_{3} \leq v t
$$

\section{HARMONIC IN TIME SOLUTIONS}

Throughout this section we consider that the end boundary data are given by

$$
\tilde{u}_{s}\left(x_{1}, x_{2}, t\right)=\tilde{v}_{s}\left(x_{1}, x_{2}\right) e^{i \omega t}, \quad \tilde{\tau}\left(x_{1}, x_{2}, t\right)=\widetilde{T}\left(x_{1}, x_{2}\right) e^{i \omega t}
$$

where $\omega$ is a positive constant, $i=\sqrt{-1}$ is the imaginary unit and $\tilde{v}_{s}\left(x_{1}, x_{2}\right)$ and $\widetilde{T}\left(x_{1}, x_{2}\right)$ are prescribed smooth functions. These data lead to a harmonic vibration inside the cylinder having the form

$$
u_{s}\left(x_{1}, x_{2}, x_{3}, t\right)=v_{s}\left(x_{1}, x_{2}, x_{3}\right) e^{i \omega t}, \quad \tau\left(x_{1}, x_{2}, x_{3}, t\right)=T\left(x_{1}, x_{2}, x_{3}\right) e^{i \omega t}
$$

whose amplitude $\left(v_{s}, T\right)$ satisfies the following differential system

$$
\begin{aligned}
& c_{11} v_{1,11}+c_{66} v_{1,22}+c_{55} v_{1,33}+\left(c_{12}+c_{66}\right) v_{2,21}+\left(c_{13}+c_{55}\right) v_{3,31} \\
& \quad-i \omega M_{11} T_{, 1}-i \omega M_{12} T_{, 2}+\rho \omega^{2} v_{1}=0 \\
& \left(c_{12}+c_{66}\right) v_{1,12}+c_{66} v_{2,11}+c_{22} v_{2,22}+c_{44} v_{2,33}+\left(c_{23}+c_{44}\right) v_{3,32} \\
& \quad-i \omega M_{12} T_{, 1}-i \omega M_{22} T_{, 2}+\rho \omega^{2} v_{2}=0 \\
& \left(c_{13}+c_{55}\right) v_{1,13}+\left(c_{23}+c_{44}\right) v_{2,23}+c_{55} v_{3,11}+c_{44} v_{3,22} \\
& \quad+c_{33} v_{3,33}-i \omega M_{33} T_{, 3}+\rho \omega^{2} v_{3}=0 \\
& \frac{1}{\theta_{0}}\left(K_{11} T_{, 11}+2 K_{12} T_{, 12}+K_{22} T_{, 22}+K_{33} T_{, 33}\right) \\
& \quad-i \omega M_{11} v_{1,1}-i \omega M_{12}\left(v_{1,2}+v_{2,1}\right)-i \omega M_{22} v_{2,2}-i \omega M_{33} v_{3,3}+\omega^{2} \frac{c}{\theta_{0}} T=0
\end{aligned}
$$

subjected to the lateral and end boundary conditions

$$
v_{s}(x)=0, \quad T(x)=0, \quad x \in \pi \cup D(L)
$$

and the base boundary conditions

$$
v_{s}\left(x_{1}, x_{2}, 0\right)=\tilde{v}_{s}\left(x_{1}, x_{2}\right), \quad T\left(x_{1}, x_{2}, 0\right)=\widetilde{T}\left(x_{1}, x_{2}\right), \quad\left(x_{1}, x_{2}\right) \in D(0)
$$


When a semi-infinite cylinder will be considered then the boundary condition (57) has to be considered only for $x \in \pi$.

In what follows we are interested by the spatial behavior of the amplitude $\left(v_{s}, T\right)$ of the considered harmonic in time vibration. To this end we assume that the elasticity tensor is strongly elliptic, $c>0, K_{33}>0$ and $K_{\alpha \beta}$ is positive definite, that is there is a positive constant $k_{0}>0$ so that

$$
K_{\alpha \beta} \xi_{\alpha} \xi_{\beta} \geq k_{0} \xi_{\alpha} \xi_{\alpha}, \quad \text { for all } \xi_{\alpha}
$$

Further, we introduce the function

$$
\begin{aligned}
K\left(x_{3}\right)= & -\int_{D\left(x_{3}\right)}\left\{\left[c_{55} v_{1,3}+\left(c_{55}-\varkappa_{2}\right) v_{3,1}\right] \bar{v}_{1}+\left[c_{55} \bar{v}_{1,3}+\left(c_{55}-\varkappa_{2}\right) \bar{v}_{3,1}\right] v_{1}\right. \\
& +\left[c_{44} v_{2,3}+\left(c_{44}-\varkappa_{1}\right) v_{3,2}\right] \bar{v}_{2}+\left[c_{44} \bar{v}_{2,3}+\left(c_{44}-\varkappa_{1}\right) \bar{v}_{3,2}\right] v_{2} \\
& +\left[\left(c_{13}+\varkappa_{2}\right) v_{1,1}+\left(c_{23}+\varkappa_{1}\right) v_{2,2}+c_{33} v_{3,3}\right] \bar{v}_{3} \\
& +\left[\left(c_{13}+\varkappa_{2}\right) \bar{v}_{1,1}+\left(c_{23}+\varkappa_{1}\right) \bar{v}_{2,2}+c_{33} \bar{v}_{3,3}\right] v_{3} \\
& \left.+i \omega M_{33}\left(\bar{T} v_{3}-T \bar{v}_{3}\right)+\frac{1}{\theta_{0}} K_{33}\left(T \bar{T}_{, 3}+T_{, 3} \bar{T}\right)\right\} d a, \quad x_{3} \in[0, L]
\end{aligned}
$$

where a bar superposed over a quantity represents the complex conjugate of that quantity and the parameters $\varkappa_{1}$ and $\varkappa_{2}$ are at our disposal.

By direct differentiation with respect to $x_{3}$ in (60) and then by using the differential system (56) and the lateral boundary condition given in (57), we obtain

$$
\begin{aligned}
-\frac{d K}{d x_{3}}\left(x_{3}\right)= & 2 \int_{D\left(x_{3}\right)}\left[c_{11} v_{1,1} \bar{v}_{1,1}+c_{22} v_{2,2} \bar{v}_{2,2}+c_{33} v_{3,3} \bar{v}_{3,3}+c_{66}\left(v_{1,2} \bar{v}_{1,2}+v_{2,1} \bar{v}_{2,1}\right)\right. \\
& +c_{55}\left(v_{1,3} \bar{v}_{1,3}+v_{3,1} \bar{v}_{3,1}\right)+c_{44}\left(v_{2,3} \bar{v}_{2,3}+v_{3,2} \bar{v}_{3,2}\right) \\
& +\left(c_{12}+\varkappa_{3}\right)\left(\bar{u}_{1,1} u_{2,2}+u_{1,1} \bar{u}_{2,2}\right)+\left(c_{13}+\varkappa_{2}\right)\left(\bar{u}_{1,1} u_{3,3}+u_{1,1} \bar{u}_{3,3}\right) \\
& +\left(c_{23}+\varkappa_{1}\right)\left(\bar{u}_{2,2} u_{3,3}+u_{2,2} \bar{u}_{3,3}\right)+\left(c_{66}-\varkappa_{3}\right)\left(\bar{u}_{1,2} u_{2,1}+u_{1,2} \bar{u}_{2,1}\right) \\
& +\left(c_{44}-\varkappa_{1}\right)\left(\bar{u}_{2,3} u_{3,2}+u_{2,3} \bar{u}_{3,2}\right)+\left(c_{55}-\varkappa_{2}\right)\left(\bar{u}_{1,3} u_{3,1}+u_{1,3} \bar{u}_{3,1}\right) \\
& +\frac{1}{\theta_{0}}\left(K_{\alpha \beta} T_{, \alpha} \bar{T}_{, \beta}+K_{33} T_{, 3} \bar{T}_{, 3}\right)-\omega^{2}\left(\rho v_{s} \bar{v}_{s}+\frac{c}{\theta_{0}} T \bar{T}\right) \\
& \left.+i \omega M_{\alpha \beta}\left(\bar{T} v_{\alpha, \beta}-T \bar{v}_{\alpha, \beta}\right)+i \omega M_{33}\left(\bar{T} v_{3,3}-T \bar{v}_{3,3}\right)\right] d a
\end{aligned}
$$

with $\varkappa_{3}$ a positive parameter at our disposal and where an identity of type (27) was also used. Then, by an appropriate choice of the parameters $\varkappa_{1}, \varkappa_{2}$ and $\varkappa_{3}$, as in the above section, we find

$$
\begin{gathered}
-\frac{d K}{d x_{3}}\left(x_{3}\right) \geq 2 \int_{D\left(x_{3}\right)}[ \\
\mu_{m} v_{r, s} \bar{v}_{r, s}+\frac{1}{\theta_{0}}\left(K_{\alpha \beta} T_{, \alpha} \bar{T}_{, \beta}+K_{33} T_{, 3} \bar{T}_{, 3}\right)-\omega^{2}\left(\rho v_{s} \bar{v}_{s}+\frac{c}{\theta_{0}} T \bar{T}\right) \\
\left.+i \omega M_{\alpha \beta}\left(\bar{T} v_{\alpha, \beta}-T \bar{v}_{\alpha, \beta}\right)+i \omega M_{33}\left(\bar{T} v_{3,3}-T \bar{v}_{3,3}\right)\right] d a
\end{gathered}
$$


In view of the lateral boundary condition given in (57) we can write

$$
\begin{aligned}
& \lambda \int_{D\left(x_{3}\right)} v_{1} \bar{v}_{1} d a \leq \int_{D\left(x_{3}\right)} v_{1, \alpha} \bar{v}_{1, \alpha} d a \\
& \lambda \int_{D\left(x_{3}\right)} v_{2} \bar{v}_{2} d a \leq \int_{D\left(x_{3}\right)} v_{2, \alpha} \bar{v}_{2, \alpha} d a \\
& \lambda \int_{D\left(x_{3}\right)} v_{3} \bar{v}_{3} d a \leq \int_{D\left(x_{3}\right)} v_{3, \alpha} \bar{v}_{3, \alpha} d a \\
& \lambda \int_{D\left(x_{3}\right)} T \bar{T} d a \leq \int_{D\left(x_{3}\right)} T_{, \alpha} \bar{T}_{, \alpha} d a
\end{aligned}
$$

where $\lambda$ is the lowest eigenvalue in the two-dimensional clamped membrane problem for the cross section $D$. By using these estimates and the Schwarz and arithmeticgeometric mean inequalities, we obtain

$$
\begin{aligned}
& \left|\int_{D\left(x_{3}\right)}\left[i \omega M_{\alpha \beta}\left(\bar{T} v_{\alpha, \beta}-T \bar{v}_{\alpha, \beta}\right)+i \omega M_{33}\left(\bar{T} v_{3,3}-T \bar{v}_{3,3}\right)\right] d a\right| \\
& \quad \leq \omega \int_{D\left(x_{3}\right)}\left[\frac{M \sqrt{\theta_{0}}}{\sqrt{\lambda k_{0} \mu_{m}}}\left(\frac{k_{0}}{\theta_{0}} T_{, \alpha} \bar{T}_{, \alpha}+\mu_{m} v_{\alpha, \beta} \bar{v}_{\alpha, \beta}\right)+\frac{\left|M_{33}\right| \sqrt{\theta_{0}}}{\sqrt{\lambda k_{0} \mu_{m}}}\left(\frac{k_{0}}{\theta_{0}} T_{, \alpha} \bar{T}_{, \alpha}+\mu_{m} v_{3,3} \bar{v}_{3,3}\right)\right] d a
\end{aligned}
$$

where $M=\left(M_{\alpha \beta} M_{\alpha \beta}\right)^{1 / 2}$. On the basis of the relations (63) and (64), from (62) we get

$$
\begin{aligned}
-\frac{d K}{d x_{3}}\left(x_{3}\right) \geq & 2 \int_{D\left(x_{3}\right)}\left[\left(\mu_{m}-\frac{M \sqrt{\theta_{0} \mu_{m}}}{\sqrt{\lambda k_{0}}} \omega-\frac{\rho}{\lambda} \omega^{2}\right) v_{\alpha, \beta} \bar{v}_{\alpha, \beta}+\left(\mu_{m}-\frac{\rho}{\lambda} \omega^{2}\right) v_{3, \alpha} \bar{v}_{3, \alpha}\right. \\
& \left.+\mu_{m}\left(1-\frac{\left|M_{33}\right| \sqrt{\theta_{0}}}{\sqrt{\lambda k_{0} \mu_{m}}} \omega\right) v_{3,3} \bar{v}_{3,3}+\mu_{m} v_{\alpha, 3} \bar{v}_{\alpha, 3}\right] d a \\
& +2 \int_{D\left(x_{3}\right)}\left[\frac{k_{0}}{\theta_{0}}-\sqrt{\frac{k_{0}}{\lambda \theta_{0} \mu_{m}}}\left(M+\left|M_{33}\right|\right) \omega-\frac{c}{\lambda \theta_{0}} \omega^{2}\right] T_{, \alpha} \bar{T}_{, \alpha} d a \\
& +2 \int_{D\left(x_{3}\right)} \frac{1}{\theta_{0}} K_{33} T_{, 3} \bar{T}_{, 3} d a
\end{aligned}
$$

Now we set

$$
\omega^{*}=\min \left(\omega_{1}, \omega_{2}\right)
$$

where

$$
\begin{aligned}
& \omega_{1}=\min \left(-\frac{M}{2 \rho} \sqrt{\frac{\lambda \theta_{0} \mu_{m}}{k_{0}}}+\frac{\lambda}{2 \rho} \sqrt{\frac{M^{2} \theta_{0} \mu_{m}}{\lambda k_{0}}+4 \mu_{m} \frac{\rho}{\lambda}}, \sqrt{\frac{\lambda \mu_{m}}{\rho}}, \frac{\sqrt{\lambda k_{0} \mu_{m}}}{\left|M_{33}\right| \sqrt{\theta_{0}}}\right) \\
& \omega_{2}=\frac{\lambda \theta_{0}}{2 c}\left[-\sqrt{\frac{k_{0}}{\lambda \theta_{0} \mu_{m}}}\left(M+\left|M_{33}\right|\right)+\sqrt{\frac{k_{0}}{\lambda \theta_{0} \mu_{m}}\left(M+\left|M_{33}\right|\right)^{2}+\frac{4 c k_{0}}{\lambda \theta_{0}^{2}}}\right]
\end{aligned}
$$


Further we assume that

$$
0<\omega<\omega^{*}
$$

so that we have

$$
\frac{d K}{d x_{3}}\left(x_{3}\right) \leq 0 \text { for all } x_{3} \in[0, L]
$$

On the other hand, from the end boundary conditions expressed in (57) and relation (60) we deduce that

$$
K(L)=0
$$

and hence (69) implies that

$$
K\left(x_{3}\right) \geq 0 \text { for all } x_{3} \in[0, L]
$$

Furthermore, from (60) we get

$$
K\left(x_{3}\right) \leq \int_{D\left(x_{3}\right)}\left(\chi_{1} v_{\alpha, \beta} \bar{v}_{\alpha, \beta}+\chi_{2} v_{3, \alpha} \bar{v}_{3, \alpha}+\chi_{3} v_{\alpha, 3} \bar{v}_{\alpha, 3}+\chi_{4} v_{3,3} \bar{v}_{3,3}+\chi_{5} T_{, \alpha} \bar{T}_{, \alpha}+\chi_{6} T_{, 3} \bar{T}_{, 3}\right) d a
$$

where

$$
\begin{aligned}
& \chi_{1}=\frac{1}{\sqrt{\lambda}} \max \left(c_{55}+\sqrt{c_{11} c_{33}}, c_{44}+\sqrt{c_{22} c_{33}}\right) \\
& \chi_{2}=\frac{1}{\sqrt{\lambda}}\left(\max \left(c_{55}, c_{44}\right)+c_{33}+\sqrt{c_{11} c_{33}}+\sqrt{c_{22} c_{33}}\right)+\frac{\omega}{\lambda}\left|M_{33}\right| \sqrt{\frac{\theta_{0} \mu_{m}}{k_{0}}} \\
& \chi_{3}=\frac{1}{\sqrt{\lambda}} \max \left(c_{55}, c_{44}\right), \quad \chi_{4}=\frac{1}{\sqrt{\lambda}} c_{33} \\
& \chi_{5}=\frac{\omega}{\lambda}\left|M_{33}\right| \sqrt{\frac{k_{0}}{\theta_{0} \mu_{m}}}+\sqrt{\frac{K_{33} k_{0}}{\lambda \theta_{0}^{2}}}, \quad \chi_{6}=\frac{K_{33}}{\theta_{0}} \sqrt{\frac{K_{33}}{k_{0} \lambda}}
\end{aligned}
$$

Concluding, from the relations (65) and (72) we get the following first order differential inequality

$$
K\left(x_{3}\right) \leq-v_{1} \frac{d K}{d x_{3}}\left(x_{3}\right) \text { for all } x_{3} \in[0, L]
$$

where

$$
v_{1}=\max \left(\frac{\chi_{1}}{\Lambda_{1}}, \frac{\chi_{2}}{\Lambda_{2}}, \frac{\chi_{3}}{\Lambda_{3}}, \frac{\chi_{4}}{\Lambda_{4}}, \frac{\chi_{5}}{\Lambda_{5}}, \frac{\chi_{6}}{\Lambda_{6}}\right)
$$


and

$$
\begin{aligned}
& \Lambda_{1}=2\left(\mu_{m}-\frac{M \sqrt{\theta_{0} \mu_{m}}}{\sqrt{\lambda k_{0}}} \omega-\frac{\rho}{\lambda} \omega^{2}\right), \quad \Lambda_{2}=2\left(\mu_{m}-\frac{\rho}{\lambda} \omega^{2}\right) \\
& \Lambda_{3}=2 \mu_{m}, \quad \Lambda_{4}=2 \mu_{m}\left(1-\frac{\left|M_{33}\right| \sqrt{\theta_{0}}}{\sqrt{\lambda k_{0} \mu_{m}}} \omega\right) \\
& \Lambda_{5}=2\left[\frac{k_{0}}{\theta_{0}}-\sqrt{\frac{k_{0}}{\lambda \theta_{0} \mu_{m}}}\left(M+\left|M_{33}\right|\right) \omega-\frac{c}{\lambda \theta_{0}} \omega^{2}\right], \quad \Lambda_{6}=\frac{2}{\theta_{0}} K_{33}
\end{aligned}
$$

By direct integration into (74) we obtain the estimate

$$
0 \leq K\left(x_{3}\right) \leq K(0) e^{-\frac{x_{3}}{v_{1}}} \text { for all } x_{3} \in[0, L]
$$

provided the frequency of vibration satisfies condition (68).

Let us discuss further the case of a semi-infinite cylinder (that is the case when $L \rightarrow \infty)$. Since $K(\cdot)$ is a non-increasing function, it follows that there are the following only two possibilities: (i) $K\left(x_{3}\right) \geq 0$ for all $x_{3} \in[0, \infty)$, or (ii) there exists $x_{3}^{*} \in(0, \infty)$ so that $K\left(x_{3}^{*}\right)<0$.

Let us first assume that (i) $K\left(x_{3}\right) \geq 0$ for all $x_{3} \in[0, \infty)$. Then, by the procedure used for finite cylinder we are led to the spatial decay estimate (77). We note that in this case the volume energetic measure

$$
\begin{aligned}
E\left(x_{3}\right)= & 2 \int_{B\left(x_{3}\right)}\left[c_{11} v_{1,1} \bar{v}_{1,1}+c_{22} v_{2,2} \bar{v}_{2,2}+c_{33} v_{3,3} \bar{v}_{3,3}+c_{66}\left(v_{1,2} \bar{v}_{1,2}+v_{2,1} \bar{v}_{2,1}\right)\right. \\
& +c_{55}\left(v_{1,3} \bar{v}_{1,3}+v_{3,1} \bar{v}_{3,1}\right)+c_{44}\left(v_{2,3} \bar{v}_{2,3}+v_{3,2} \bar{v}_{3,2}\right) \\
& +\left(c_{12}+\varkappa_{3}\right)\left(\bar{v}_{1,1} v_{2,2}+v_{1,1} \bar{v}_{2,2}\right)+\left(c_{13}+\varkappa_{2}\right)\left(\bar{v}_{1,1} v_{3,3}+v_{1,1} \bar{v}_{3,3}\right) \\
& +\left(c_{23}+\varkappa_{1}\right)\left(\bar{v}_{2,2} v_{3,3}+v_{2,2} \bar{v}_{3,3}\right)+\left(c_{66}-\varkappa_{3}\right)\left(\bar{v}_{1,2} v_{2,1}+v_{1,2} \bar{v}_{2,1}\right) \\
& +\left(c_{44}-\varkappa_{1}\right)\left(\bar{v}_{2,3} v_{3,2}+v_{2,3} \bar{v}_{3,2}\right)+\left(c_{55}-\varkappa_{2}\right)\left(\bar{v}_{1,3} v_{3,1}+v_{1,3} \bar{v}_{3,1}\right) \\
& +\frac{1}{\theta_{0}}\left(K_{\alpha \beta} T_{, \alpha} \bar{T}_{, \beta}+K_{33} T_{, 3} \bar{T}_{, 3}\right)-\omega^{2}\left(\rho v_{s} \bar{v}_{s}+\frac{c}{\theta_{0}} T \bar{T}\right)+i \omega M_{\alpha \beta}\left(\bar{T} v_{\alpha, \beta}-T \bar{v}_{\alpha, \beta}\right) \\
& \left.+i \omega M_{33}\left(\bar{T} v_{3,3}-T \bar{v}_{3,3}\right)\right] d v \geq 0
\end{aligned}
$$

exists and it is finite.

Let us now discuss the second case, that is we assume that $K\left(x_{3}^{*}\right)<0$ for some $x_{3}^{*} \in(0, \infty)$. Since $K(\cdot)$ is a non-increasing function on $[0, \infty)$, it follows that we have

$$
K\left(x_{3}\right) \leq K\left(x_{3}^{*}\right)<0 \text { for all } x_{3} \in\left[x_{3}^{*}, \infty\right)
$$

and now we obtain

$$
-K\left(x_{3}\right) \leq-v_{1} \frac{d K}{d x_{3}}\left(x_{3}\right) \text { for all } x_{3} \in\left[x_{3}^{*}, \infty\right)
$$


By integration in (80) we get the following estimate

$$
-K\left(x_{3}\right) \geq-K\left(x_{3}^{*}\right) e^{\frac{x_{3}-x_{3}^{*}}{v_{1}}} \text { for all } x_{3} \in\left[x_{3}^{*}, \infty\right)
$$

and we have to observe that in this case the volume energetic measure $E\left(x_{3}\right)$ is infinite. These results can be embodied in the following Phragmén-Lindelöf alternative result: In the context of a semi-infinite cylinder we have the following alternative: a) for the amplitudes having a finite volume energetic measure $E\left(x_{3}\right)$, the $K\left(x_{3}\right)$, as given by (60), is a measure which decays spatially faster than the exponential $e^{-\frac{x_{3}}{v_{1}}}$ for all $x_{3} \in[0, \infty)$, or b) for the amplitudes having an infinite volume energetic measure $E\left(x_{3}\right)$, the $-K\left(x_{3}\right)$ grows spatially faster than the exponential $e^{\frac{x_{3}-x_{3}^{*}}{v_{1}}}$ for all $x_{3} \in\left[x_{3}^{*}, \infty\right)$, provided the frequency $\omega$ is lower that the critical value $\omega^{*}$ defined by (66).

\section{REFERENCES}

1. D. S. Chandrasekharaiah, Hyperbolic Thermoelasticity: A Review of Recent Literature, Appl. Mech. Rev., vol. 51, pp. 705-729, 1998.

2. R. B. Hetnarski and J. Ignaczak, Generalized Thermoelasticity, J. Thermal Stresses, vol. 22, pp. 451-470, 1999.

3. R. B. Hetnarski and J. Ignaczak, Nonclassical Dynamical Thermoelasticity, Int. J. Solids Struct., vol. 37, pp. 215-224, 2000.

4. A. E. Green and P. M. Naghdi, Thermoelasticity Without Energy Dissipation, J. Elasticity, vol. 31, pp. 189-208, 1993.

5. A. E. Green and P. M. Naghdi, On Thermodynamics and the Nature of the Second Law, Proc. Roy. Soc. London A, vol. 357, pp. 253-270, 1977.

6. A. E. Green and P. M. Naghdi, A Unified Procedure for Contruction of Theories of Deformable Media. I. Classical Continuum Physics, II. Generalized Continua, III. Mixtures of Interacting Continua, Proc. Royal Society London A, vol. 448, pp. 335-356, pp. 357-377, pp. 379-388, 1995.

7. L. Nappa, Spatial Decay Estimates for the Evolution Equations of Linear Thermoelasticity Without Energy Dissipation, J. Thermal Stresses, vol. 21, pp. 581-592, 1998.

8. R. Quintanilla, On the Spatial Behavior in Thermoelasticity Without Energy Dissipation, J. Thermal Stresses, vol. 22, pp. 213-224, 1999.

9. D. S. Chandrasekharaiah, A Note on the Uniqueness of Solution in the Linear Theory of Thermoelasticity Without Energy Dissipation, J. Elasticity, vol. 43, pp. 279-283, 1996.

10. D. Iesan, On the Theory of Thermoelasticity Without Energy Dissipation. J. Thermal Stresses, vol. 21, pp. 295-307, 1998.

11. R. Quintanilla, On Existence in Thermoelasticity Without Energy Dissipation, J. Thermal Stresses, vol. 25, pp. 195-202, 2002.

12. R. Quintanilla, Damping of End Effects in a Thermoelastic Theory, Appl. Math. Letters, vol. 14, pp. 137-141, 2001.

13. R. Quintanilla, Convergence and Structural Stability in Thermoelasticity, Appl. Math. Computation, vol. 135, pp. 287-300, 2003.

14. R. Quintanilla and B. Straughan, Growth and Uniqueness in Thermoelasticity, Proc. Royal Society London A, vol. 456, pp. 1419-1429, 2000.

15. R. Quintanilla and B. Straughan, Energy Bounds for some Non-Standard Problems in Thermoelasticity, Proc. Royal Society London A, vol. 461, pp. 1147-1162, 2005. 
16. J. N. Flavin, R. J. Knops, and L. E. Payne, Energy Bounds in Dynamical Problems for a Semi-infinite elastic beam, in G. Eason and R. W. Ogden (eds.), Elasticity: Mathematical Methods and Applications, pp. 101-112, Ellis-Horwood (Wiley), Chichester, 1990.

17. S. Chiriţă and R. Quintanilla, On Saint-Venant's Principle in Linear Elastodynamics, J. Elasticity, vol. 42, pp. 201-215, 1996.

18. S. Chiriţă and M. Ciarletta, Time-Weighted Surface Power Function Method for the Study of Spatial Behaviour in Dynamics of Continua, Euro. J. Mech., A/Solids, vol. 18, pp. 915-933, 1999.

19. S. Chiriţă, Spatial Decay Estimates for Solutions Describing Harmonic Vibrations in a Thermoelastic Cylinder, J. Thermal Stresses, vol. 18, pp. 421-436, 1995.

20. S. Chiriţă, Spatial Behavior in the Strongly Elliptic Anisotropic Thermoelastic Materials, J. Thermal Stresses, vol. 30, pp. 859-873, 2007.

21. S. Chiriţă, A. Danescu, and M. Ciarletta, On the Strong Ellipticity of the Anisotropic Linearly Elastic Materials, J. Elasticity, vol. 87, pp. 1-27, 2007. 
Copyright of Journal of Thermal Stresses is the property of Taylor \& Francis Ltd and its content may not be copied or emailed to multiple sites or posted to a listserv without the copyright holder's express written permission. However, users may print, download, or email articles for individual use. 